EPJ Web of Conferences 41, 01005 (2013)

DOI: $10.1051 /$ epjconf/20134101005

(C) Owned by the authors, published by EDP Sciences, 2013

\title{
Attosecond physics at a nanoscale metal tip
}

\author{
Michael Krüger ${ }^{1}$, Markus Schenk ${ }^{1}$, Michael Förster ${ }^{1}$, Sebastian Thomas ${ }^{1}$, Georg Wachter ${ }^{2}$, Christoph \\ Lemell $^{2}$, Joachim Burgdörfer ${ }^{2}$, Peter Hommelhoff ${ }^{1, \text { a }}$ \\ 1 Max Planck Institute of Quantum Optics, H.-Kopfermann-Str. 1, D-85748 Garching, Germany, EU \\ 2 Institute for Theoretical Physics, Vienna University of Technology, Wiedner Hauptstr. 8-10, A-1040 \\ Vienna, Austria, EU
}

\begin{abstract}
With few-cycle laser oscillator pulses at $800 \mathrm{~nm}$ we observe strong-field and attosecond physics phenomena in electron spectra recorded at a nanoscale tungsten tip. We observe the rescattering plateau as well as a strong carrier-envelope phase dependence of the spectra. We model the results with the semiclassical three-step model as well as with time-dependent density functional theory.
\end{abstract}

\section{Introduction}

Attosecond physics is based on controlling photo-emitted electrons with the optical electric field. Of particular importance is the recollision process, in which an electron is driven back to its parent matter. There, it can recombine, scatter elastically ("rescattering") or inelastically. We have observed several hallmarks of attosecond physics at a nanoscale tungsten tip: In section 2 we report on matterwave interference in the time-energy domain, which exhibits a strong carrier-envelope (C-E) phase dependence. In section 3 we detail our investigations on the rescattering effect at a solid metal tip. Section 4 concludes with an outlook.

\section{Carrier-envelope phase effects at a tip}

We focus $\sim 6.5 \mathrm{fs}$ pulses of a C-E phase stable laser oscillator (Venteon) on a tungsten tip. The tip is electrochemically etched and ends in an approximate hemisphere with a radius of curvature in the range of $5 \ldots 20 \mathrm{~nm}$. The laser beam is tightly focused down to $\sim 2.2 \mu \mathrm{m}$ and impinges on the tip in the plane perpendicular to the tip pointing direction, with the polarization vector parallel to the latter. With $240 \mathrm{~nJ}$ pulse energy we observe spectra like the one shown in Fig. 1 (a): they show a direct part and a plateau part. Section 3 will show that the plateau arises from elastic rescattering of emitted electrons at the metal tip, in great analogy to what is known from atoms and molecules in the gas phase [1].

In the direct part we observe above-threshold photon orders [2], which we will not discuss here. In the plateau part, photon orders are visible, too. However, if we record the spectra as a function of the C-E phase (Fig. 1 (b, c)), the photon orders almost fully disappear for certain settings of the C-E phase. We explain this behavior with the three-step model, adapted for a metal [3]: In the first step, electrons are being emitted with the ADK rate [4], even though the Keldysh parameter $\gamma \approx 2$, including field enhancement. (With other emission rates we observe a qualitatively similar behavior, however, the data do not as nicely match [5].) In the propagation step, we only consider events that undergo elastic scattering at the tip, within a single optical cycle after emission. The quantum mechanical phase is accumulated, and finally all trajectories that lead to the same final kinetic energy are summed coherently. The results are shown in Fig. 1 (d, e). Evidently, depending on the C-E phase the electrons can

\footnotetext{
a e-mail: peter.hommelhoff@mpq.mpg.de
} unrestricted use, distribution, and reproduction in any medium, provided the original work is properly cited. 

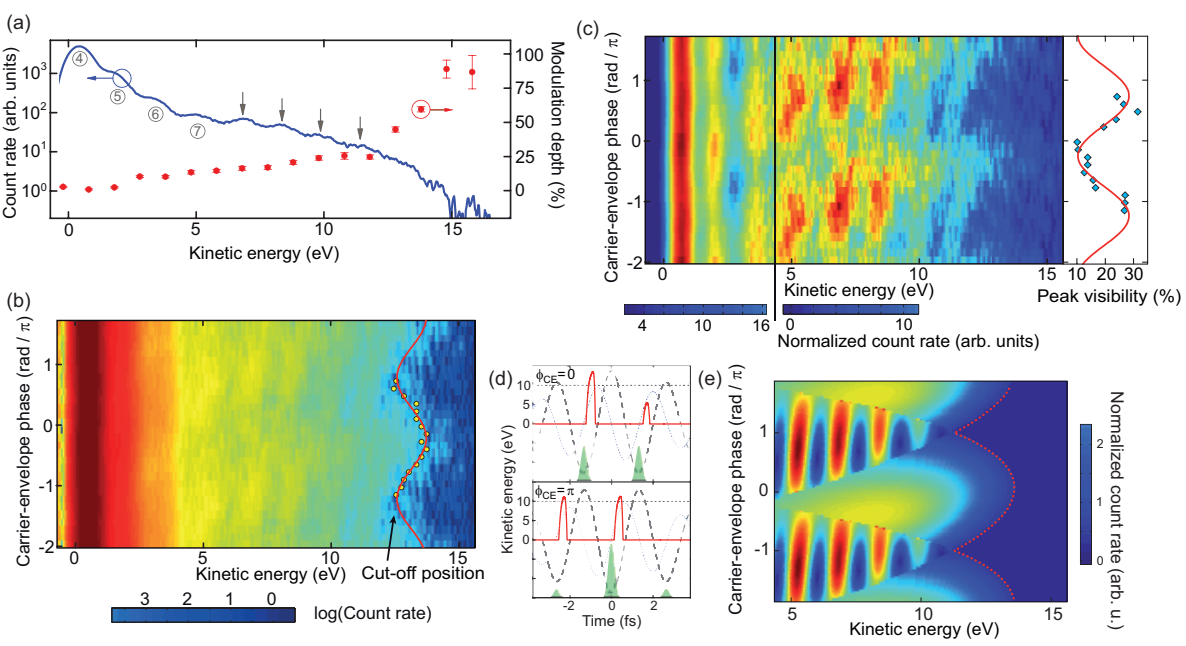

Fig. 1. (a) Spectrum with the direct part $(0 \ldots 5 \mathrm{eV})$ and the plateau part $(5 \ldots 13 \mathrm{eV})$. The numbers in circles give the photon orders, 4 being the lowest free one. (b) Same as in (a), but as a function of the C-E phase. The dots and the red line show the cut-off position, i.e., the highest kinetic energies reached. (c) Data of (b), but with an exponential slope in each, the direct and the plateau part, subtracted to enhance visibility of phase effects. Photon orders (red islands) are visible for certain C-E phase settings, whereas they almost fully disappear for other settings. The diamonds indicate the visibility of the photon orders. (d) Results of the three-step model: final kinetic energy of an electron as a function of the emission time. (e) Three-step model data treated like the data in (c). Clearly, regions of strong modulation exist, and other regions that are almost flat as a function of the kinetic energy, much like in (c). The three step model data hence allow explaining what is responsible for the appearance and disappearance of photon orders: Photon orders appear if two windows in time exists at which electrons are emitted; photon order visibility ceases if only one emission window in time contributes, as can be inferred from (d). In close analogy to Young's double slit experiment, we here observe a double or a single slit in time, leading to interference in the energy domain. Sharp boundaries exist in (e) because of the semiclassical nature of the process.

gain high kinetic energies during one or two optical cycles. In the first case, the largest kinetic energy results, and a flat spectrum is visible; in the second case strong interference fringes are visible and a comparably small maximum kinetic energy is reached. Hence, the three-step model can nicely explain the data qualitatively, and also quantitatively surprisingly well: peak electric fields of $10.4 \mathrm{GV} / \mathrm{m}$ are needed at the tip, from which we infer a field enhancement factor of $\sim 6$, roughly as expected. The unmodulated plateau part hints at an electron emission duration of 450 attoseconds (Fourier limit of cut-off region).

\section{Rescattering at a tip}

While the three step model allows interpretation of the data astonishingly well, it is by no means clear that it is applicable to metals. In order to unmask the effects that take place at a metal surface, we have invoked a model applicable to metals: we employ a 1-dimensional time-dependent density functional theory (TDDFT) quantum simulation. Details of the model are given in [6,7], results are shown in Fig. 2.

Fig. 2 (a) shows the change of the electron density on a logarithmic color scale. The rescattering process is directly visualized in the center of the laser pulse (indicated in the lower panel): electron density is driven about $10 \ldots 20$ atomic units (a.u.) $\approx 0.5 \ldots 1 \mathrm{~nm}$ out of the metal, before it is being pushed back towards the metal surface, matching the classical excursion of $0.3 \mathrm{~nm}$ quite well. Note that for these parameters it is not yet required to take into account the steep field gradient in front of the tip [8]. 

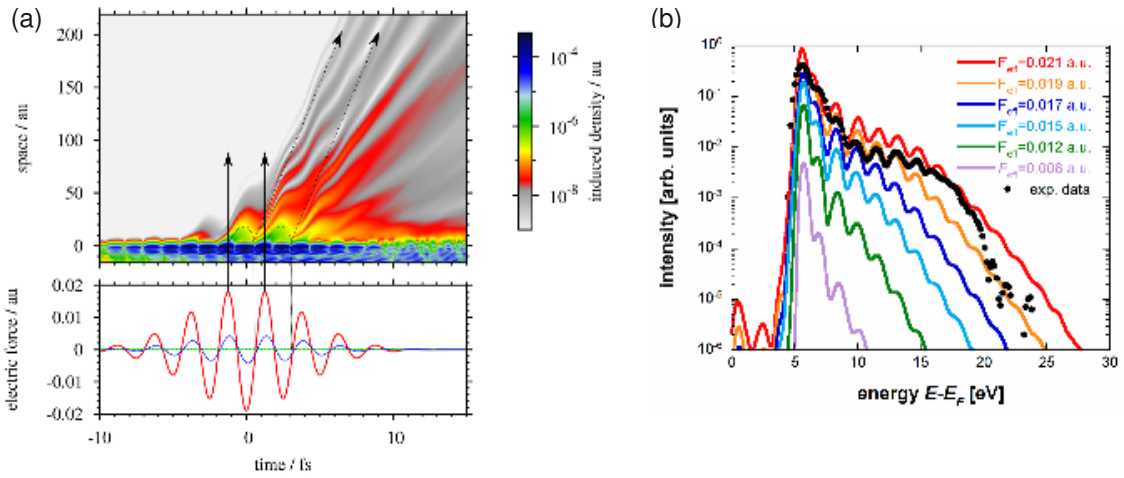

Fig. 2. (a) Result of the TDDFT calculation: electron density as a function of time and distance from the surface (1-d). Clearly, electronic density is being pulled out of the metal by the laser field, and subsequently pushed back, directly showing rescattering at the metal. (b) Spectra obtained from the TDDFT model for various peak field strengths (solid lines), and a experimental spectrum (dots) for comparison. Clearly, TDDFT results and data agree fairly well, not only in the plateau but also in the direct part. Figures taken from $[6,7]$.

Fig. 2 (a) displays a number of spectra for different laser peak fields obtained from the TDDFT model, together with experimental data. Clearly, a peak electric field strength of $0.02 \mathrm{au}$, corresponding to a laser intensity of $1.4 \times 10^{13} \mathrm{~W} / \mathrm{cm}^{2}$, matches the data best. Interestingly, both direct and plateau part are nicely reproduced by the theory model, indicating the suitability of the model.

\section{Outlook}

In summary, we have shown that the most important attosecond science techniques can now be applied to solids, namely to nanometric metal tips. New fields of research may emerge: The rescattered electron might probe the surface of the tip and so might yield information of ultrafast surface processes; attosecond low-energy electron diffraction might result, however, here higher return energies $(\sim 100 \mathrm{eV})$ will be necessary to obtain diffraction orders. With the natural length scale of the tip in the nanometer range, near-field optics lies at the center of interest. The electron might serve as a means to tomographically map the optical near-field, with a resolution in the sub-nm scale. Collective electron excitations (plasmons) can be probed. Further, the carrier electric field can fully switch on and off the high energy part of the current - on the attosecond time scale. Hence, with a drain structure nearby, the tip might serve as the source in an attosecond field effect transistor. The combination of single atom tips [9] with these techniques could allow interesting comparisons between bound and free atoms.

\section{References}

1. G.G. Paulus, W. Nicklich, H. Xu, P. Lambropoulos, H. Walther, Phys. Rev. Lett. 72, 2851 (1994)

2. M. Schenk, M. Krüger, P. Hommelhoff, Phys. Rev. Lett. 105, 257601 (2010)

3. P.B. Corkum, Phys. Rev. Lett. 71, 1994 (1993)

4. M. Ammosov, N. Delone, V. Krainov, Sov. Phys. JETP 64, 1191 (1986)

5. M. Krüger, M. Schenk, M. Förster, P. Hommelhoff, J. Phys. B 45, 074006 (2012)

6. G. Wachter, C. Lemell, J. Burgdörfer, M. Schenk, M. Krüger, P. Hommelhoff, Phys. Rev. B 86, $035402(2012)$

7. M. Krüger, M. Schenk, P. Hommelhoff, G. Wachter, C. Lemell, J. Burgdörfer (2012), to be published in New J. Phys.

8. G. Herink, D.R. Solli, M. Gulde, C. Ropers, Nature 483, 190 (2012)

9. P. Hommelhoff, C. Kealhofer, A. Aghajani-Talesh, Y.R. Sortais, S.M. Foreman, M.A. Kasevich, Ultramicroscopy 109, 423 (2009) 